PROCEEDINGS OF THE

AMERICAN MATHEMATICAL SOCIETY

Volume 130, Number 10, Pages 3051-3065

S 0002-9939(02)06525-5

Article electronically published on March 18, 2002

\title{
ON THE COMPLEXITY OF THE DESCRIPTION OF *-ALGEBRA REPRESENTATIONS BY UNBOUNDED OPERATORS
}

\author{
LYUDMILA TUROWSKA
}

(Communicated by David R. Larson)

\begin{abstract}
We study the complexity of the problem to describe, up to unitary equivalence, representations of $*$-algebras by unbounded operators on a Hilbert space. A number of examples are developed in detail.
\end{abstract}

Representations of algebras and $*$-algebras by bounded and unbounded operators on a Hilbert space have many applications in various branches of analysis and mathematical physics and is a powerful tool for the study of algebras itself. Often it is important to estimate how complicated the problem of describing representations (up to an equivalence) is. In the theory of representations of algebras the representation problem is considered to be extremely difficult (wild) if it "contains" the classical unsolved problem of describing up to a similarity a pair of matrices without relations (see $[\mathrm{DF}]$ ). For $*$-algebras the complexity of the structure of their representations by bounded operators on a Hilbert space was studied in [KS] (see also OS [S]). The authors introduced a quasiorder $\succ$ (majorization) of $*$-algebras with respect to how difficult their representations are and proved that the enveloping $C^{*}$-algebra, $C^{*}\left(\mathcal{F}_{2}\right)$, of the free group with two generators, majorizes any finitely generated $*$-algebra showing that the problem of describing $*$-representations (up to unitary equivalence) of a $*$-algebra $\mathfrak{A}$ such that $\mathfrak{A} \succ C^{*}\left(\mathcal{F}_{2}\right)$ is difficult (such an algebra $\mathfrak{A}$ is called $*$-wild). Note that for a unital $C^{*}$-algebra $\mathfrak{A}, \mathfrak{A} \succ C^{*}\left(\mathcal{F}_{2}\right)$ is equivalent to $\mathfrak{A} / J \simeq M_{n}(\mathbb{C}) \otimes C^{*}\left(\mathcal{F}_{2}\right)$ where $J$ is a $C^{*}$-ideal of $\mathfrak{A}$ and $n \in \mathbb{N}([\mathbf{O S}]$ ). Boundedness of $*$-representations is essential for their consideration.

In the present paper we study the complexity of $*$-algebras representations by unbounded operators on a Hilbert space. Unbounded representations occur, for example, in the representation theory of non-compact Lie algebras, non-compact quantum groups and quantum algebras, etc. (see [JSW, OS, Wor1, Wor2] and references therein). For instance, the $*$-algebra with two selfadjoint generators $p, q$ and the canonical (Heisenberg) commutation relation $p q-q p=i$ of the quantum mechanics does not have any representations by bounded operators but it has well known unbounded ones: $p=i \frac{d}{d x}, q=x$ on $L_{2}(\mathbb{R}, d x)$. That the structure of unbounded representations can be very complicated for *-algebras is already evident from [S, Chapter 9.4] and [ST1]: the problem of describing unbounded

Received by the editors June 12, 2000 and, in revised form, November 28, 2000 and May 31, 2001.

2000 Mathematics Subject Classification. Primary 46K10, 46L05, 47L60; Secondary 16G60.

Key words and phrases. *-algebras, *-representations, unbounded operators, *-wildness.

(C)2002 American Mathematical Society 
*-representations for the *-algebra $\mathbb{C}\left[x_{1}, x_{2}\right]$ of all polynomials in commuting selfadjoint elements $x_{1}, x_{2}$ "contains" as a subproblem the problem of classification up to unitary equivalence all representations of $C^{*}\left(\mathcal{F}_{2}\right)$.

In Section 3, developing [KS, $\mathrm{OS}$, we give a definition of complicated (*-wild) classes of $*$-representations by unbounded operators. An invaluable tool for our study is the notion of unbounded elements which generate a $C^{*}$-algebra introduced and studied by Woronowicz [Wor2. We prove that the class of bounded representations of a unital $*$-algebra $\mathfrak{A}$ is complicated (*-wild) iff the algebra $\mathfrak{A}$ is $*$-wild in the sense of [KS] (Theorem [4). Similar to [KS], we show that the class of representations which "arises from" representations of a $C^{*}$-algebra $\mathfrak{A}$ is complicated iff $\mathfrak{A} / J \simeq C B(H) \otimes C^{*}\left(\mathcal{F}_{2}\right)$, where $C B(H)$ are now compact operators on a Hilbert space $H$ (Theorem 5 ). From this we derive that integrable representations of finite-dimensional Lie algebras, i.e. infinitesimal representations $d U$ of unitary representations $U$ of the corresponding Lie group, are not $*$-wild (Corollary 1 ).

In Section 4, we study, from the point of view developed in Section 3, the complexity of representations by unbounded operators of some $*$-algebras. The first example is devoted to non-integrable representations of the polynomial *algebra $\mathbb{C}\left[x_{1}, x_{2}\right]$. The second example deals with the $*$-algebra $\mathfrak{A}=\mathbb{C}\left\langle x_{1}, x_{2}\right|$ $\left.\left[x_{1},\left[x_{1}, x_{2}\right]\right]=0, x_{i}^{*}=x_{i}, i=1,2\right\rangle$. Note that operators satisfying the double commutation relations were studied in [Dal] in the framework of abstract hyperbolic equations. In the third example we discuss a class of unbounded representations generated by idempotents with zero sum.

We attempt to give sufficient background references for all concepts involved. For the basic definitions and notions of the theory of representations of $*$-algebras and $C^{*}$-algebras we refer the reader to $[\underline{D}, \operatorname{Ped}] \underline{S}$. Throughout the paper $H$ is a separable Hilbert space, $C B(H)$ and $B(H)$ denote the algebra of compact and respectively bounded operators on $H$. For $*$-algebras $A$ and $B$ the algebraic tensor product of $A$ and $B$ is denoted by $A \odot B$. We write $\operatorname{Rep}(A)$ for the category of $*$-representations of $A$, with bounded non-degenerate representations as objects and intertwining operators as morphisms. Recall that a $*$-representation $\pi$ of a *-algebra $A$ on a Hilbert space $H$ is non-degenerate if $\pi(A) H$ is dense in $H$.

\section{1. *-WILD ALGEBRAS}

We follow [KS] in introducing the notion of $*$-wild algebras (see also [OS]). Within this section we assume that all $*$-algebras are unital and representations of $*$-algebras are unital $*$-homomorphisms into $B(H)$.

Let $\mathfrak{A}$ be a $*$-algebra. If $\psi: \mathfrak{A} \rightarrow M_{n}(\mathbb{C}) \otimes C^{*}\left(\mathcal{F}_{2}\right), n \in \mathbb{N}$, is a unital *homomorphism of the *-algebra $\mathfrak{A}$ and the $C^{*}$-algebra $M_{n}(\mathbb{C}) \otimes C^{*}\left(\mathcal{F}_{2}\right)$ (= $M_{n}\left(C^{*}\left(\mathcal{F}_{2}\right)\right)$, then there is a natural way to construct the functor $F_{\psi}$ : $\operatorname{Rep}\left(C^{*}\left(\mathcal{F}_{2}\right)\right) \rightarrow \operatorname{Rep}(\mathfrak{A}):$

- $F_{\psi}(\pi)=(i d \otimes \pi) \circ \psi$, for any $\pi \in \operatorname{Rep}\left(C^{*}\left(\mathcal{F}_{2}\right)\right)$,

- $F_{\psi}(\alpha)=I \otimes \alpha$ for any operator $\alpha$ intertwining $\pi_{1}, \pi_{2} \in \operatorname{Rep}\left(C^{*}\left(\mathcal{F}_{2}\right)\right)$.

( $I$ is the identity operator in $B\left(\mathbb{C}^{n}\right)$.)

Definition 1. A $*$-algebra $\mathfrak{A}$ is called $*$-wild if there exist $n \in \mathbb{N}$ and a $*$-homomorphism $\psi: \mathfrak{A} \rightarrow M_{n}(\mathbb{C}) \otimes C^{*}\left(\mathcal{F}_{2}\right)$ such that the functor $F_{\psi}: \operatorname{Rep}\left(C^{*}\left(\mathcal{F}_{2}\right)\right) \rightarrow \operatorname{Rep}(\mathfrak{A})$ is full. 
In order to verify that the functor $F_{\psi}$ is full one has to show that for representations $\pi_{1}, \pi_{2} \in \operatorname{Rep}\left(C^{*}\left(\mathcal{F}_{2}\right)\right)$ in $H_{1}$ and $H_{2}$ respectively, an operator $A$ intertwines the representations $F_{\psi}\left(\pi_{1}\right)$ and $F_{\psi}\left(\pi_{2}\right)$ iff $A=I \otimes \alpha$, where $I$ is the identity operator in $B\left(\mathbb{C}^{n}\right)$ and $\alpha$ intertwines the representations $\pi_{1}, \pi_{2}$. It follows from the definition that two representations $\pi_{1}, \pi_{2}$ of $C^{*}\left(\mathcal{F}_{2}\right)$ are unitarily equivalent iff so are the representations $F_{\psi}\left(\pi_{1}\right), F_{\psi}\left(\pi_{2}\right)$ of $\mathfrak{A}$, a representation $\pi$ of $C^{*}\left(\mathcal{F}_{2}\right)$ is irreducible iff so is $F_{\psi}(\pi)$. Thus the unitary classification problem of all representations of $\mathfrak{A}$ contains, as a subproblem, the problem of unitary classification of all representations of $C^{*}\left(\mathcal{F}_{2}\right)$. As it was noticed in the introduction, $C^{*}\left(\mathcal{F}_{2}\right)$ majorizes any finitely-generated $*$-algebra and the unitary classification of all representations of $C^{*}\left(\mathcal{F}_{2}\right)$ contains, as a subproblem, the problem of unitary classification of any affine $*$-algebra. An example of a $*$-wild algebra is $\mathfrak{S}_{2}=\mathbb{C}\left\langle a, b \mid a=a^{*}, b=b^{*}\right\rangle$. For $C^{*}$-algebras we have the following:

Theorem $1([\mathrm{OS}])$. A $C^{*}$-algebra $\mathfrak{A}$ is $*$-wild if and only if there exist a $C^{*}$-ideal $J \subset \mathfrak{A}$ and $n \in \mathbb{N}$ such that $\mathfrak{A} / J \simeq M_{n}(\mathbb{C}) \otimes C^{*}\left(\mathcal{F}_{2}\right)$.

Remark 1. It follows from the above theorem that a unital $C^{*}$-algebra $\mathfrak{A}$ is $*$-wild iff there exists a $*$-epimorphism $\psi: \mathfrak{A} \rightarrow M_{n}\left(C^{*}\left(\mathcal{F}_{2}\right)\right)$ and if, in particular, $\mathfrak{A}$ is generated by some elements $T_{1}, \ldots, T_{n}$, i.e. $\mathfrak{A}$ is the closure of algebraic combinations of $1, T_{1}, \ldots, T_{n}, T_{1}^{*}, \ldots, T_{n}^{*}$, then $\psi\left(T_{1}\right), \ldots \psi\left(T_{n}\right)$ generate $M_{n}\left(C^{*}\left(\mathcal{F}_{2}\right)\right)$.

For other results and examples of $*$-wild algebras we refer the reader to [OS].

\section{2. $C^{*}$-Algebras Generated By A Finite NUMBER OF AFFILIATED ELEMENTS}

In order to generalise the notion of $*$-wild algebra to include representations by unbounded operators on a Hilbert space, we need unbounded elements which are "related" to a $C^{*}$-algebra. The notion of a $C^{*}$-algebra generated by unbounded affiliated elements was introduced and investigated by S. L. Woronowicz (Wor2, see also [Wor1]). We recall here some definitions and facts from Wor2 which will be used in the sequence.

Let $H$ be a Hilbert space, $C^{*}(H)$ the set of separable non-degenerate $C^{*}$ subalgebras of $B(H)$, and $A \in C^{*}(H)$. The set of all multipliers, $M(A)$, of $A$ is defined by

$$
M(A)=\{a \in B(H) \mid a b, b a \in A, \text { for any } b \in A\} .
$$

Let $T$ be a closed operator acting on a Hilbert space $H$. We say that $T$ is affiliated with $A \in C^{*}(H)$ if the $z$-transform $z_{T}=T\left(I+T^{*} T\right)^{-1 / 2}$ of $T$ belongs to $M(A), z_{T}^{*} z_{T} \leq I$ and $\left(I-z_{T}^{*} z_{T}\right) A$ is dense in $A$. We write $T \eta A$. The set of all elements affiliated with $A$ will be denoted by $A^{\eta}$. T is a linear mapping acting on $A$ with the domain $D(T)=\left(I-z_{T}^{*} z_{T}\right)^{1 / 2} A . D \subseteq D(T)$ is a core of $T$ if $T$ coincides with the closure of $\left.T\right|_{D}$. One should distinguish between $D(T)$ and the domain of $T$ as an operator acting on a Hilbert space which will be denoted by $\mathcal{D}(T)$.

Let $A$ be a $C^{*}$-algebra, $B \in C^{*}(H)$. The set of morphisms, $\operatorname{Mor}(A, B)$, consists of all $\pi \in \operatorname{Rep}(A, H)$ such that $\pi(A) B$ is dense in $B$, where $\operatorname{Rep}(A, H)$ is the set of all non-degenerate representations of $A$ on $H$. In particular, $\operatorname{Mor}(A, C B(H))=$ $\operatorname{Rep}(A, H)$. If $\varphi \in \operatorname{Mor}(A, B)$, then $\varphi$ can be uniquely extended to a mapping from $A^{\eta}$ to $B^{\eta}$.

The notions of multiplier algebra, affiliated elements are independent of the choice of embedding of $C^{*}$-algebras into $B(H)$ (see Wor2]). 
Let $A$ be a $C^{*}$-algebra and $T_{1}, \ldots, T_{n}$ be elements affiliated with $A$. We say that $A$ is generated by $T_{1}, \ldots, T_{n}$ if for any Hilbert space $H, B \in C^{*}(H)$ and any $\pi \in \operatorname{Rep}(A, H)$ the condition $\pi\left(T_{i}\right) \eta B, i=1, \ldots, n$, implies $\pi \in \operatorname{Mor}(A, B)$. We will use the following sufficient condition for a $C^{*}$-algebra $A$ to be generated by elements affiliated with $A$.

Theorem 2. Let $A$ be a $C^{*}$-algebra and $T_{1}, \ldots, T_{n}$ be elements affiliated with $A$. The subset of $M(A)$ composed of all elements of the form $\left(I+T_{i}^{*} T_{i}\right)^{-1}$ and $\left(I+T_{i} T_{i}^{*}\right)^{-1}, i=1, \ldots, n$, will be denoted by $\Gamma$. Assume that:

1. $T_{1}, \ldots, T_{n}$ separate representations of $A:$ if $\varphi_{1}, \varphi_{2}$ are different elements of $\operatorname{Rep}(A, H)$, then $\varphi_{1}\left(T_{i}\right) \neq \varphi_{2}\left(T_{i}\right)$ for some $i=1, \ldots, n$.

2. There exist elements $r_{1}, \ldots, r_{k} \in \Gamma$ such that the product $r_{1} \ldots r_{k} \in A$. Then $A$ is generated by $T_{1}, \ldots, T_{n}$.

Remark 2. A $C^{*}$-algebra $A$ is generated by $T_{1}, \ldots, T_{n} \eta A$ with $\left\|T_{i}\right\|<\infty$ for all $i=1, \ldots, n$, if and only if $A$ is unital and $A$ coincides with the norm closure of all algebraic combinations of $I, T_{1}, \ldots, T_{n}, T_{1}^{*}, \ldots, T_{n}^{*}$.

See [Wor2] for other discussions on $C^{*}$-algebras generated by affiliated elements.

\section{The COMPLEXITy OF UnBOUNDED REPRESENTATIONS OF *-ALGEBRAS}

In this section we shall extend the notion of a $*$-wild problem to unbounded representations. We restrict our attention to finitely presented unital $*$-algebras, i.e. $*$-algebras introduced in terms of a finite number of generators and relations (algebraic equalities imposed on the generators).

Let $\mathfrak{A}$ be a unital $*$-algebra generated by elements $t_{1}, \ldots, t_{n}, t_{1}^{*}, \ldots, t_{n}^{*}$ and relations

$$
w_{j}\left(t_{1}, \ldots, t_{n}, t_{1}^{*}, \ldots, t_{n}^{*}\right)=0, \quad j=1, \ldots, m,
$$

where $w_{j}$ are polynomials over $\mathbb{C}$ in the non-commuting variables $t_{1}, \ldots, t_{n}, t_{1}^{*}, \ldots$, $t_{n}^{*}$ and 1.

A family of closed operators $T_{1}, \ldots, T_{n}, T_{1}^{*}, \ldots, T_{n}^{*}$ on a Hilbert space $H$ is called a representation, $\pi$, of $\mathfrak{A}$ if there exists a dense domain, $\mathcal{D}$, such that $\mathcal{D}$ is invariant with respect to all operators of the family, $\mathcal{D}$ is a core for $T_{i}, T_{i}^{*}, i=1, \ldots, n$, and the relations

$$
w_{j}\left(T_{1}, \ldots, T_{n}, T_{1}^{*}, \ldots, T_{n}^{*}\right) \varphi=0, \quad j=1, \ldots, m,
$$

hold for any $\varphi \in \mathcal{D}$ (with the identity operator instead of 1 ). We write $\pi\left(t_{i}\right)=$ $T_{i}$. We denote by $\operatorname{Rep}_{\text {unb }}(\mathfrak{A})$ the category of unbounded representations of $\mathfrak{A}$. The objects of $\operatorname{Rep}_{\text {unb }}(\mathfrak{A})$ are representations defined above and the morphisms of $\operatorname{Rep}_{\text {unb }}(\mathfrak{A})$ are bounded operators $C \in B(H, \tilde{H})$ intertwining representations $\pi$ and $\tilde{\pi}$ which act on Hilbert spaces $H$ and $\tilde{H}$ respectively, i.e. $C T_{i} \subseteq \tilde{T}_{i} C, C T_{i}^{*} \subseteq \tilde{T}_{i}^{*} C$, $i=1, \ldots, n$ (we write $C \in I(\pi, \tilde{\pi})$ ). We say that two representations, $\pi_{1}$ and $\pi_{2}$, are unitarily equivalent if there exists a unitary operator of $H\left(\pi_{1}\right)$ onto $H\left(\pi_{2}\right)$ such that $U \in I\left(\pi_{1}, \pi_{2}\right)$ and $U^{-1} \in I\left(\pi_{2}, \pi_{1}\right)$. In this case we write $\pi_{1} \simeq \pi_{2}$.

Proving that the problem of unitary classification of bounded representations of a $*$-algebra $A$ is $*$-wild we construct a unital $*$-homomorphism $\psi: A \rightarrow M_{n}(\mathbb{C}) \otimes$ $C^{*}\left(\mathcal{F}_{2}\right), n \in \mathbb{N}$, which generates a full functor $F_{\psi}: \operatorname{Rep}\left(C^{*}\left(\mathcal{F}_{2}\right)\right) \rightarrow \operatorname{Rep}(\mathfrak{A})$. In order to include unbounded representations we shall first replace the above *homomorphism by a "*-homomorphism" into the set of affiliated elements 
$\left(C B(H) \otimes C^{*}\left(\mathcal{F}_{2}\right)\right)^{\eta}$, where $C B(H)$ is the $C^{*}$-algebra of a compact operator on a Hilbert space (not necessarily finite-dimensional) and $C B(H) \otimes C^{*}\left(\mathcal{F}_{2}\right)$ is the $C^{*}$ algebra obtained by the completion of the algebraic tensor product $C B(H) \odot C^{*}\left(\mathcal{F}_{2}\right)$ in a $C^{*}$-norm (it is known that it does not depend on norm).

Namely, let $\psi$ be a unital mapping from $\left(1, t_{1}, \ldots, t_{n}\right)$ to $\left(C B(H) \otimes C^{*}\left(\mathcal{F}_{2}\right)\right)^{\eta}$ such that there exists a dense linear subset $D$ of $C B(H) \otimes C^{*}\left(\mathcal{F}_{2}\right)$ satisfying the following conditions:

- $D \subseteq D\left(\psi\left(t_{i}\right)\right), D \subseteq D\left(\psi\left(t_{i}\right)^{*}\right), \psi\left(t_{i}\right) D \subseteq D, \psi\left(t_{i}\right)^{*} D \subseteq D$,

- $w_{j}\left(\psi\left(t_{1}\right), \ldots, \psi\left(t_{n}\right), \psi\left(t_{1}\right)^{*}, \ldots, \psi\left(t_{n}\right)^{*}\right) a=0, \quad j=1, \ldots, m, \quad a \in D$,

- $D$ is a core for $\psi\left(t_{1}\right), \ldots, \psi\left(t_{n}\right), \psi\left(t_{1}\right)^{*}, \ldots, \psi\left(t_{n}\right)^{*}$.

In the sequel, whenever we write a $*$-homomorphism $\psi$ from $\mathfrak{A}$ to $(C B(H) \otimes$ $\left.C^{*}\left(\mathcal{F}_{2}\right)\right)^{\eta}$, we mean a unital mapping $\psi:\left(1, t_{1}, \ldots, t_{n}\right) \rightarrow\left(C B(H) \otimes C^{*}\left(\mathcal{F}_{2}\right)\right)^{\eta}$ satisfying the above conditions.

As before, the mapping $\psi$ generates a functor $F_{\psi}: \operatorname{Rep}\left(C^{*}\left(\mathcal{F}_{2}\right)\right) \rightarrow \operatorname{Rep}_{\text {unb }}(\mathfrak{A})$ :

- $F_{\psi}(\pi)\left(t_{i}\right)=(i d \otimes \pi)\left(\psi\left(t_{i}\right)\right)$ for any representation $\pi \in \operatorname{Rep}\left(C^{*}\left(\mathcal{F}_{2}\right)\right)$, where $i d \otimes \pi$ is the unique extension to the affiliated elements,

- $F_{\psi}(\alpha)=I \otimes \alpha$ for any $\alpha$ intertwining $\pi_{1}, \pi_{2} \in \operatorname{Rep}\left(C^{*}\left(\mathcal{F}_{2}\right)\right)$.

If $\pi$ is a representation of $C^{*}\left(\mathcal{F}_{2}\right)$ on a Hilbert space $H(\pi)$, then $F_{\psi}(\pi)\left(t_{i}\right)$, $i=1, \ldots, n$, define a representation of $\mathfrak{A}$ with $\mathcal{D}=\{(i d \otimes \pi)(D) \varphi, \varphi \in H \otimes H(\pi)\}$, $\overline{\mathcal{D}}=H \otimes H(\pi)$.

Consider the following two properties of the mapping $\psi: \mathfrak{A} \rightarrow\left(C B(H) \otimes C^{*}\left(\mathcal{F}_{2}\right)\right)^{\eta}$ : (P.1.) the corresponding functor $F_{\psi}$ is full, and (P.2.) $\psi\left(t_{1}\right), \ldots, \psi\left(t_{n}\right)$ generate $C B(H) \otimes C^{*}\left(\mathcal{F}_{2}\right)$ as affiliated elements. We will see below that in the case of unital $C^{*}$-algebra, $\mathcal{A}$, the second condition (not the first one) implies *-wildness of $\mathcal{A}$ and the result of Theorem 1 partially explains this (see also Remark 1). Theorem 3 shows that (P.2) implies (P.1) but the inverse is false (see Remark 5).

Theorem 3. Let $\psi: \mathfrak{A} \rightarrow\left(C B(H) \otimes C^{*}\left(\mathcal{F}_{2}\right)\right)^{\eta}$ be a *-homomorphism in the sense defined above. Assume that $\psi\left(t_{1}\right), \ldots, \psi\left(t_{n}\right)$ generate $C B(H) \otimes C^{*}\left(\mathcal{F}_{2}\right)$. Then the corresponding functor $F_{\psi}: \operatorname{Rep}\left(C^{*}\left(\mathcal{F}_{2}\right)\right) \rightarrow \operatorname{Rep}_{\text {unb }}(\mathfrak{A})$ is full.

Proof. To prove the statement it is enough to show that given an operator $C \in$ $B(H)$, a representation $\pi \in \operatorname{Rep}\left(C^{*}\left(\mathcal{F}_{2}\right)\right)$ such that $C F_{\psi}(\pi)\left(t_{i}\right) \subseteq F_{\psi}(\pi)\left(t_{i}\right) C$, $C F_{\psi}(\pi)\left(t_{i}\right)^{*} \subseteq F_{\psi}(\pi)\left(t_{i}\right)^{*} C$, we have $C=I \otimes \alpha$, where $\alpha \in I(\pi, \pi)$.

We first show that $C$ commutes with $(i d \otimes \pi)(a)$ for any $a \in C B(H) \otimes C^{*}\left(\mathcal{F}_{2}\right)$. Define the following set:

$$
\mathfrak{B}_{C}=\left\{a \in M(\mathfrak{B}): \begin{array}{l}
C(i d \otimes \pi)(a)=(i d \otimes \pi)(a) C, \\
C(i d \otimes \pi)\left(a^{*}\right)=(i d \otimes \pi)\left(a^{*}\right) C .
\end{array}\right\}
$$

Here $\mathfrak{B}=C B(H) \otimes C^{*}\left(\mathcal{F}_{2}\right) . \mathfrak{B}_{C}$ is a $C^{*}$-subalgebra of $M(\mathfrak{B})$. Let us now show that $\mathfrak{B}_{C}=M(\mathfrak{B})$. We will use Wor2, Proposition 2.2], an analogue of the StoneWeierstrass Theorem.

(1) $\mathfrak{B}_{C}$ is non-empty, since the $z$-transforms $z_{\psi\left(t_{i}\right)}, z_{\psi\left(t_{i}\right)}^{*}, i=1, \ldots, n$, belong to $\mathfrak{B}_{C}$.

(2) $\mathfrak{B}_{C}$ separates representations of $\mathfrak{B}$. Indeed, let $\pi_{1}, \pi_{2}$ be two different representations of $\mathfrak{B}$. Assuming that $\pi_{1}(q)=\pi_{2}(q)$ for any $q \in \mathfrak{B}_{C}$ we get $\pi_{1}\left(z_{\psi\left(t_{i}\right)}\right)=\pi_{2}\left(z_{\psi\left(t_{i}\right)}\right)$ which implies $\pi_{1}\left(\psi\left(t_{i}\right)\right)=\pi_{2}\left(\psi\left(t_{i}\right)\right)$ for any $i=1, \ldots, n$. Since $\psi\left(t_{1}\right), \ldots, \psi\left(t_{n}\right)$ generate $\mathfrak{B}$, we get $\pi_{1}=\pi_{2}$. A contradiction. 
(3) $\mathfrak{B}_{C}$ is strictly closed. In fact, let $\left\{x_{\lambda}\right\} \in \mathfrak{B}_{C}$ strictly converge to $x$, i.e. $\left\|a\left(x_{\lambda}-x\right)\right\| \rightarrow 0$ and $\left\|\left(x_{\lambda}-x\right) a\right\| \rightarrow 0$ for any $a \in \mathfrak{B}$. If $\pi \in \operatorname{Rep}(\mathfrak{B}, H)$, we get $\left\|\pi\left(x_{\lambda} a\right)-\pi(x a)\right\| \rightarrow 0$ and

$$
\left(\pi\left(x_{\lambda}\right)-\pi(x)\right) \pi(a) \varphi \rightarrow 0, \quad a \in \mathfrak{B}, \varphi \in H .
$$

Since $\pi$ is a non-degenerate representation of $\mathfrak{B}$, the set $\{\pi(a) \varphi: a \in \mathfrak{B}, \varphi \in H\}$ is dense in $H$ and any strictly convergent sequence is bounded, we deduce that $\left(\pi\left(x_{\lambda}\right)-\pi(x)\right) \varphi \rightarrow 0, \varphi \in H$. Therefore, if $C \pi\left(x_{\lambda}\right)=\pi\left(x_{\lambda}\right) C$ we get $C \pi(x)=\pi(x) C$ and $x \in \mathfrak{B}_{C}$.

According to [Wor2, Proposition 2.2], (1), (2), (3) imply $\mathfrak{B}_{C}=M(\mathfrak{B})$ and hence $[C,(i d \otimes \pi)(a)]=0$ for any $a \in C B(H) \otimes C^{*}\left(\mathcal{F}_{2}\right)$. This gives $C=I \otimes \alpha$, where $[\alpha, \pi(a)]=0$ for any $a \in C^{*}\left(\mathcal{F}_{2}\right)$, and hence the functor $F_{\psi}$ is full.

In the sequel we shall consider classes, $R$, of representations from $\operatorname{Rep}_{\text {unb }}(\mathfrak{A})$ which are closed with respect to the direct sum and taking subrepresentations, i.e. $R$ satisfies the following conditions: a) if $\pi_{1} \in R$ and $\pi_{1} \simeq \pi_{2}$, then $\pi_{2} \in R$; b) if $\left(\pi_{\lambda}\right)_{\lambda \in \Lambda}$ is a family of representations from $R$, where $\Lambda$ is a countable set of indexes, then $\bigoplus_{\lambda \in \Lambda} \pi_{\lambda} \in R$; c) if $\pi_{1} \oplus \pi_{2} \in R$, then $\pi_{i} \in R, i=1,2$.

Definition 2. Let $\mathfrak{A}$ be a unital $*$-algebra generated by $t_{1}, \ldots, t_{n}, t_{1}^{*}, \ldots, t_{n}^{*}$ and relations (11). We say that a class $R \subset \operatorname{Rep}_{\text {unb }}(\mathfrak{A})$ is $*$-wild if there exists a *homomorphism $\psi: \mathfrak{A} \rightarrow\left(C B(H) \otimes C^{*}\left(\mathcal{F}_{2}\right)\right)^{\eta}$ such that $(i d \otimes \pi)(\psi)$ belongs to $R$ for any $\pi \in \operatorname{Rep}\left(C^{*}\left(\mathcal{F}_{2}\right)\right)$ and $\psi\left(t_{1}\right), \ldots, \psi\left(t_{n}\right)$ generate the $C^{*}$-algebra $C B(H) \otimes$ $C^{*}\left(\mathcal{F}_{2}\right)$.

Theorem 4. The class of bounded representations of $\mathfrak{A}$ is *-wild if and only if $\mathfrak{A}$ is $*$-wild.

Proof. Assume that there exists a $*$-homomorphism $\psi: \mathfrak{A} \rightarrow\left(C B(H) \otimes C^{*}\left(\mathcal{F}_{2}\right)\right)^{\eta}$ such that the operators $(i d \otimes \pi)\left(\psi\left(t_{i}\right)\right)$ are bounded for any $\pi \in \operatorname{Rep}\left(C^{*}\left(\mathcal{F}_{2}\right)\right)$, and $\psi\left(t_{1}\right), \ldots, \psi\left(t_{n}\right)$ generate $\mathfrak{B}=C B(H) \otimes C^{*}\left(\mathcal{F}_{2}\right)$. Clearly, $\psi\left(t_{i}\right), i=1, \ldots, n$, are bounded. It follows from [Wor2, Example 1] that $\mathfrak{B}$ is unital and coincides with the norm closure of all algebraic combinations of $I, \psi\left(t_{1}\right), \ldots, \psi\left(t_{n}\right), \psi\left(t_{1}\right)^{*}, \ldots, \psi\left(t_{n}\right)^{*}$. This implies that $H$ is finite-dimensional and $\psi$ is a $*$-homomorphism from $\mathfrak{A}$ to $C B(H) \otimes C^{*}\left(\mathcal{F}_{2}\right)$. Moreover, by Theorem [3, the functor generated by $\psi$ is full which means that the *-algebra $\mathfrak{A}$ is $*$-wild.

Conversely, suppose that $\mathfrak{A}$ is $*$-wild. Then there exists a $*$-homomorphism $\psi: \mathfrak{A} \rightarrow C B(H) \otimes C^{*}\left(\mathcal{F}_{2}\right)$ with $\operatorname{dim} H<\infty$. Let $\tilde{\mathfrak{B}}$ be the norm closure of the set of all algebraic combinations of $I, \psi\left(t_{1}\right), \ldots, \psi\left(t_{n}\right), \psi\left(t_{1}\right)^{*}, \ldots, \psi\left(t_{n}\right)^{*}$. Then $\tilde{\mathfrak{B}}$ is a $C^{*}$-subalgebra of $C B(H) \otimes C^{*}\left(\mathcal{F}_{2}\right)$. Since $\mathfrak{A}$ is $*$-wild, it is not difficult to see that $\pi(\psi(\mathfrak{A}))^{\prime}=\pi\left(C B(H) \otimes C^{*}\left(\mathcal{F}_{2}\right)\right)^{\prime}$ for any representation $\pi$ of $C B(H) \otimes$ $C^{*}\left(\mathcal{F}_{2}\right)$ (see, for example, the proof of [OS, Theorem 50]). This implies $\pi(\tilde{\mathfrak{B}})^{\prime}=$ $\pi\left(C B(H) \otimes C^{*}\left(\mathcal{F}_{2}\right)\right)^{\prime}$. By [OS, Lemma 14], the inclusion $i: \tilde{\mathfrak{B}} \rightarrow C B(H) \otimes C^{*}\left(\mathcal{F}_{2}\right)$ is a surjection, and hence $\tilde{\mathfrak{B}}=C B(H) \otimes C^{*}\left(\mathcal{F}_{2}\right)$. Since the $C^{*}$-algebra $C B(H) \otimes$ $C^{*}\left(\mathcal{F}_{2}\right)$ is unital, by Remark 2 , we see that $\psi\left(t_{1}\right), \ldots, \psi\left(t_{n}\right)$ generate $C B(H) \otimes$ $C^{*}\left(\mathcal{F}_{2}\right)$ in the sense of affiliated elements.

Let $\mathfrak{A}$ be a $*$-algebra generated by $t_{1}, \ldots, t_{n}, t_{1}^{*}, \ldots, t_{n}^{*}$ and relations (1). We say that a class $R \subset \operatorname{Rep}_{\text {unb }}(\mathfrak{A})$ is manageable (see Wor2]) if there exist a separable $C^{*}$-algebra $\mathfrak{B}$ (unital or non-unital) and $T_{1}, \ldots, T_{n} \eta \mathfrak{B}$ such that $\mathfrak{B}$ is generated 
by $T_{1}, \ldots, T_{n}$ and $R$ is equal to the set

$$
\left\{\pi \in \operatorname{Rep}_{\text {unb }}(\mathfrak{A}): \pi\left(t_{i}\right)=\tilde{\pi}\left(T_{i}\right) \text { for some } \tilde{\pi} \in \operatorname{Rep}(\mathfrak{B})\right\} .
$$

Example 1 ([Wor2]). Let $G$ be a connected, simply connected Lie group, $c_{k l}^{i}$, $k, l=1, \ldots, n$, be the structure constants of the Lie algebra $A$ of $G$ and the class $R$ consists of integrable representations of $A$, or equivalently families $\left(T_{1}, \ldots, T_{n}\right)$ such that $T_{1}, \ldots, T_{n}$ are skew-adjoint operators acting on a Hilbert space $H$ such that on a dense invariant domain $\left[T_{k}, T_{l}\right]=\sum_{i=1}^{n} c_{k l}^{i} T_{i}$ and $\sum_{i=1}^{n} T_{i}^{2}$ is essentially self-adjoint on the same domain. Then the class $R$ is manageable and $\mathfrak{B}=C^{*}(G)$.

Theorem 5. Let $R$ be a manageable class of *-representations of $\mathfrak{A}$. Let $\mathfrak{B}$ be the $C^{*}$-algebra defined above. Then $R$ is *-wild if and only if there exist a $C^{*}$-ideal $J$ and a Hilbert space $H$ such that

$$
\mathfrak{B} / J \simeq C B(H) \otimes C^{*}\left(\mathcal{F}_{2}\right) .
$$

Proof. Assume that $R$ is $*$-wild. Then there exists $\psi: \mathfrak{A} \rightarrow\left(C B(H) \otimes C^{*}\left(\mathcal{F}_{2}\right)\right)^{\eta}$ such that $\psi\left(t_{1}\right), \ldots, \psi\left(t_{n}\right)$ generate $C B(H) \otimes C^{*}\left(\mathcal{F}_{2}\right)$ and $(i d \otimes \pi)(\psi) \in R$ for any $\pi \in \operatorname{Rep}\left(C^{*}\left(\mathcal{F}_{2}\right)\right)$. We can assume that $C^{*}\left(\mathcal{F}_{2}\right)$ is embedded into $B\left(H_{0}\right)$. Let $\tilde{\pi} \in \operatorname{Rep}\left(C^{*}(\mathfrak{B})\right)$ such that $\psi\left(t_{i}\right)=\tilde{\pi}\left(T_{i}\right)$. Since $T_{1}, \ldots, T_{n}$ generate $\mathfrak{B}$, we get $\tilde{\pi} \in \operatorname{Mor}\left(\mathfrak{B}, C B(H) \otimes C^{*}\left(\mathcal{F}_{2}\right)\right)$. Applying [Wor2, Proposition 3.2] we conclude that $\tilde{\pi}(\mathfrak{B})=C B(H) \otimes C^{*}\left(\mathcal{F}_{2}\right)$ and hence there exists an ideal $J=\operatorname{ker} \tilde{\pi}$ such that (3) holds.

Assume now that (3) holds with an isomofism $\varphi: \mathfrak{B} / J \rightarrow C B(H) \otimes C^{*}\left(\mathcal{F}_{2}\right)$. Let $\pi$ denote the quotient map $\pi: \mathfrak{B} \rightarrow \mathfrak{B} / J$. Since $T_{1}, \ldots, T_{n}$ generate $\mathfrak{B}$ it follows from the definition that $(\varphi \circ \pi)\left(T_{1}\right), \ldots,(\varphi \circ \pi)\left(T_{n}\right)$ generate $C B(H) \otimes C^{*}\left(\mathcal{F}_{2}\right)$. Setting $\psi\left(t_{i}\right)=(\varphi \circ \pi)\left(T_{i}\right)$ we get a $*$-homomorphism from $\mathfrak{A}$ to $\left(C B(H) \otimes C^{*}\left(\mathcal{F}_{2}\right)\right)^{\eta}$ satisfying the conditions of Definition 2 Therefore the class $R$ is $*$-wild.

Corollary 1. Integrable representations of a finite-dimensional Lie algebra are not *-wild.

Proof. Assuming the class of an integrable representation of a finite-dimensional Lie algebra to be *-wild we get by Theorem 5 and Example 1 that the $C^{*}$ algebra $C^{*}(G)$ of the corresponding connected, simply connected Lie group $G$ contains a $C^{*}$-ideal $J$ such that $C^{*}(G) / J \simeq C B(H) \otimes C^{*}\left(\mathcal{F}_{2}\right)$. However $C^{*}(G)$ is nuclear (see [Pat] $)$, and therefore has only hyperfinite factor representations while $C B(H) \otimes C^{*}\left(\mathcal{F}_{2}\right)$ has non-hyperfinite representations. A contradiction.

Remark 3. If we have a $*$-homomorphism $\psi: \mathfrak{A} \rightarrow\left(C B(H) \otimes C^{*}\left(\mathcal{F}_{2}\right)\right)^{\eta}$ which generates a full functor $F_{\psi}: \operatorname{Rep}\left(C^{*}\left(\mathcal{F}_{2}\right)\right) \rightarrow R \subset \operatorname{Rep}_{\text {unb }}(\mathfrak{A})$, then the problem of unitary classification of $*$-representations $R$ of $\mathfrak{A}$ is difficult and contains as a subproblem the problem of unitary classification of all representations of $C^{*}\left(\mathcal{F}_{2}\right)$. Namely, the representation $\left((i d \otimes \pi)(\psi)\left(t_{1}\right), \ldots,(i d \otimes \pi)(\psi)\left(t_{n}\right)\right), \pi \in C^{*}\left(\mathcal{F}_{2}\right)$, is irreducible iff so is $\pi$, two such representations are unitarily equivalent iff so are the corresponding representations of $C^{*}\left(\mathcal{F}_{2}\right)$.

In Section 4 we will use the following proposition.

Proposition 1. Let $\mathfrak{B}$ be a $C^{*}$-algebra such that (3) holds, and let $\psi: \mathfrak{A} \rightarrow$ $\left(C B\left(H_{0}\right) \otimes \mathfrak{B}\right)^{\eta}$ be a unital $*$-homomorphism such that $\psi\left(t_{1}\right), \ldots, \psi\left(t_{n}\right)$ generate $C B\left(H_{0}\right) \otimes \mathfrak{B}$. Assume that the representation $\left((i d \otimes \pi)\left(\psi\left(t_{1}\right)\right), \ldots,(i d \otimes \pi)\left(\psi\left(t_{n}\right)\right)\right.$ belongs to a class $R \subset \operatorname{Rep}_{\text {unb }}(\mathfrak{A})$ for any $\pi \in \operatorname{Rep}(\mathfrak{B})$. Then $R$ is $*$-wild. 
Proof. By assumption, there exists a $*$-homomorphism $\varphi: \mathfrak{B} \rightarrow C B\left(H_{1}\right) \otimes C^{*}\left(\mathcal{F}_{2}\right)$ such that

$$
\varphi(\mathfrak{B})=C B\left(H_{1}\right) \otimes C^{*}\left(\mathcal{F}_{2}\right) .
$$

If we prove that $\left(i d_{H_{0}} \otimes \varphi\right)\left(\psi\left(t_{i}\right)\right), i=1, \ldots, n$, generate $\mathcal{A}=C B\left(H_{0}\right) \otimes C B\left(H_{1}\right) \otimes$ $C^{*}\left(\mathcal{F}_{2}\right)$, the assertion follows. Here $i d_{H}$ is the identity representation of $C B(H)$. Let $\mathcal{H}$ be a separable Hilbert space and $\pi \in \operatorname{Rep}(\mathcal{A}, \mathcal{H})$. Then there exist a unitary operator $V \in B(\mathcal{H})$ and a representation $\rho \in \operatorname{Rep}\left(C^{*}\left(\mathcal{F}_{2}\right)\right)$ such that

$$
\pi=V\left(i d_{H_{0}} \otimes i d_{H_{1}} \otimes \rho\right) V^{-1} .
$$

Let $\mathcal{C} \in C^{*}(\mathcal{H})$ and $\pi\left(\left(i d_{H_{0}} \otimes \varphi\right)\left(\psi\left(t_{i}\right)\right)\right) \eta \mathcal{C}$. Then

$$
\left(i d_{H_{0}} \otimes i d_{H_{1}} \otimes \rho\right)\left(\left(i d_{H_{0}} \otimes \varphi\right)\left(\psi\left(t_{i}\right)\right)\right) \eta V^{-1} \mathcal{C} V=: \tilde{\mathcal{C}} .
$$

Since $\tilde{\rho}:=\left(i d_{H_{1}} \otimes \rho\right)(\varphi)$ is a representation of $\mathfrak{B}, i d_{H_{0}} \otimes \tilde{\rho} \in \operatorname{Mor}\left(C B\left(H_{0}\right) \otimes \mathfrak{B}, \tilde{\mathcal{C}}\right)$. From this and (4) we conclude that $i d_{H_{0}} \otimes i d_{H_{1}} \otimes \rho \in \operatorname{Mor}(\mathcal{A}, \tilde{\mathcal{C}})$ and $\pi \in \operatorname{Mor}(\mathcal{A}, \mathcal{C})$. The proof is finished.

Remark 4. Let $R$ be a class of $\operatorname{Rep}_{u n b}(\mathfrak{A})$, and let $H$ and $H_{0}$ be separable Hilbert spaces, $\operatorname{dim} H=\infty$. We denote by $R(\mathcal{H})$ the set of all representations $\pi \in R$ acting on $\mathcal{H}=H \otimes H_{0}$. Assume that there exists a $*$-homomorphism $\psi: \mathfrak{A} \rightarrow$ $\left(C B(H) \otimes C^{*}\left(\mathcal{F}_{2}\right)\right)^{\eta}$. We shall write $R_{\psi}(\mathcal{H})$ for the subset of those representations $\pi \in R(\mathcal{H})$ which are generated by $\psi$, i.e., $R_{\psi}(\mathcal{H})=\left\{(\pi)\left(\psi\left(t_{1}\right)\right), \ldots,(\pi)\left(\psi\left(t_{n}\right)\right)\right.$, $\left.\pi \in \operatorname{Rep}\left(C B(H) \otimes C^{*}\left(\mathcal{F}_{2}\right)\right)\right\}$. Then $R(\mathcal{H})$ and $R_{\psi}(\mathcal{H})$ are complete subsets of $\left(\mathbb{C}^{N} \otimes C B(\mathcal{H})\right)^{\eta}$. Denote by $C_{\infty}\left(R_{\psi}(\mathcal{H})\right)$ and $C_{\infty}(R(\mathcal{H}))$ the $C^{*}$-algebras of all "vanishing at infinity" continuous operator functions defined on $R_{\psi}(\mathcal{H})$ and $R(\mathcal{H})$ respectively (see [Wor2]). Then one can show that if $R$ is $*$-wild, then $C B(H) \otimes$ $C^{*}\left(\mathcal{F}_{2}\right) \simeq C_{\infty}\left(R_{\psi}(\mathcal{H})\right)$ and if $R(\mathcal{H})$ is "locally compact", then $*$-wildness of $R$ is equivalent to $C_{\infty}(R(\mathcal{H})) / J \simeq C B(H) \otimes C^{*}\left(\mathcal{F}_{2}\right)$, where $J$ is a $C^{*}$-ideal of $C_{\infty}(R(\mathcal{H}))$.

\section{EXAMPLES}

4.1. Unbounded representations of $\mathbb{C}\left[x_{1}, x_{2}\right]$. Let $\mathfrak{A}=\mathbb{C}\left[x_{1}, x_{2}\right]$ be a unital *-algebra of all polynomials in two commuting hermitian generators $x_{1}, x_{2}$. It is known that any irreducible integrable representation $\pi$ of the algebra is onedimensional. Recall that for $\mathbb{C}\left[x_{1}, x_{2}\right]$ a representation $\pi=\left(X_{1}=X_{1}^{*}, X_{2}=X_{2}^{*}\right)$ is integrable if the spectral projections of $X_{1}$ and $X_{2}$ commute. It was shown by Schmüdgen $[\mathrm{S}]$ that for any properly infinite von Neumann algebra $\mathcal{N}$ on a separable Hilbert space there exists a non-integrable $*$-representation $\rho=\left(X_{1}=X_{1}^{*}, X_{2}=\right.$ $X_{2}^{*}$ ) of $\mathfrak{A}$ such that the spectral projections of these operators generate the von Neumann algebra $\mathcal{N}$. The result which was given without proof in [ST1] is that the classification of such representations "contains as a subproblem" the problem of unitary classification of representations of the $*$-algebra $\mathfrak{S}_{2}=\mathbb{C}\langle a, b| a=a^{*}, b=$ $\left.b^{*}\right\rangle$. We repeat relevant material from [ST1].

Let $\alpha, \beta, \varepsilon_{1}, \varepsilon_{2}>0$. Consider the set $\mathcal{R}$ of all representations $\pi$ of $\mathfrak{S}_{2}$ such that $\|\pi(a)\| \leq \alpha,\|\pi(b)\| \leq \beta$ and $\pi(a) \geq \varepsilon_{1}, \pi(b) \geq \varepsilon_{2}$. Denote by $\mathfrak{B}_{\alpha, \beta}^{\varepsilon_{1}, \varepsilon_{2}}$ the completion of $\mathfrak{S}_{2} /\{z:|\|z \mid\|=0\}$ under $\||| z|| \mid=\sup \{\|\rho(z)\| ; \rho \in \mathcal{R}\}$. Consider the following construction, analogous to the one in $\left[\mathbf{S}\right.$. Consider $p, q \in M_{3}\left(\mathfrak{B}_{\alpha, \beta}^{\varepsilon_{1}, \varepsilon_{2}}\right)$ given by

$$
p=\left(\begin{array}{lll}
\lambda e_{1} & \mu a & 0 \\
\mu a & \lambda e_{1} & \mu b \\
0 & \mu b & \lambda e_{1}
\end{array}\right), \quad q=\left(\begin{array}{lll}
e_{1} & 0 & 0 \\
0 & 0 & 0 \\
0 & 0 & 0
\end{array}\right)
$$


where $\lambda, \mu \in \mathbb{R}$ are such that $1 / 2<p<3 / 4, e_{n}$ is the unit element in $M_{n}\left(\mathfrak{B}_{\alpha, \beta}^{\varepsilon_{1}, \varepsilon_{2}}\right)$. Let $w_{1}, w_{2} \in M_{2}\left(M_{3}\left(\mathfrak{B}_{\alpha, \beta}^{\varepsilon_{1}, \varepsilon_{2}}\right)\right) \simeq M_{6}\left(\mathfrak{B}_{\alpha, \beta}^{\varepsilon_{1}, \varepsilon_{2}}\right)$ be defined by

$$
w_{1}=\left(\begin{array}{cc}
i\left(e_{3}-2 q\right) & 0 \\
0 & e_{3}
\end{array}\right), \quad w_{2}=\left(\begin{array}{cc}
e_{3}-2 p & -2\left(p-p^{2}\right)^{1 / 2} \\
-2\left(p-p^{2}\right)^{1 / 2} & 2 p-e_{3}
\end{array}\right) .
$$

Since $1 / 2<p<3 / 4$, the element $w_{2}$ is well-defined.

Let $H$ be an infinite-dimensional separable Hilbert space and let $\left\{f_{k}, k \in \mathbb{Z}\right\}$ be an orthonormal basis in $H$. Let $P_{k}$ be the projection onto $\mathbb{C}\left\langle f_{k}\right\rangle$ and $v$ be the shift operator, i.e., $v f_{k}=f_{k+1}, k \in \mathbb{Z}$. We now define $v_{1}, v_{2}, v_{3} \in B(H) \odot M_{6}\left(\mathfrak{B}_{\alpha, \beta}^{\varepsilon_{1}, \varepsilon_{2}}\right)$ to be

$$
\begin{gathered}
v_{1}=v \otimes e_{6}, \quad v_{2}=v\left(I-P_{1}\right) \otimes e_{6}+v P_{1} \otimes w_{1}, \\
v_{3}=v\left(I-P_{1}-P_{2}\right) \otimes e_{6}+v P_{1} \otimes w_{1}+v P_{2} \otimes w_{2} .
\end{gathered}
$$

Finally, we define operators $U_{1}$ and $U_{2} \in B(H) \odot B(H) \odot M_{6}\left(\mathfrak{B}_{\alpha, \beta}^{\varepsilon_{1}, \varepsilon_{2}}\right) \subset B(\mathcal{H}) \odot$ $\mathfrak{B}_{\alpha, \beta}^{\varepsilon_{1}, \varepsilon_{2}}, \mathcal{H}=\bigoplus_{i=1}^{6} H \otimes H$ by

$$
U_{1}=v \otimes E, \quad U_{2}=\left(\sum_{i=-\infty}^{1} P_{i}\right) \otimes v_{1}+P_{2} \otimes v_{2}+\left(\sum_{i=3}^{+\infty} P_{i}\right) \otimes v_{3},
$$

where $E$ is the unity in $B(H) \odot M_{6}\left(\mathfrak{B}_{\alpha, \beta}^{\varepsilon_{1}, \varepsilon_{2}}\right)$.

Proposition 2. There exist self-adjoint elements $X_{1}, X_{2} \eta C B(\mathcal{H}) \otimes \mathfrak{B}_{\alpha, \beta}^{\varepsilon_{1}, \varepsilon_{2}}$ such that $U_{1}, U_{2}$ are the Cayley transforms of $X_{1}$ and $X_{2}$ respectively.

Proof. We denote by $A$ the $C^{*}$-algebra $C B(\mathcal{H}) \otimes \mathfrak{B}_{\alpha, \beta}^{\varepsilon_{1}, \varepsilon_{2}} . U_{1}, U_{2}$ are unitary elements of $M\left(C B(\mathcal{H}) \otimes \mathfrak{B}_{\alpha, \beta}^{\varepsilon_{1}, \varepsilon_{2}}\right)$. According to WN Proposition 5.1, Theorem 5.2], it suffices to show that $\left(I-U_{i}^{*}\right) A$ is dense in $A, i=1,2$. Suppose for the moment that the statement is false. Then $\left(I-U_{i}^{*}\right) A$ is a proper right ideal in $A$ and there exists a pure state on $A$ such that $f\left(\left(I-U_{i}^{*}\right) a\right)=0$, for any $a \in A$ ([D, Theorem 2.9.5]). Using the GNS procedure we can construct a representation $\pi \in \operatorname{Rep}(A, H)$ and a cyclic vector $\varphi \in H$ such that $f(a)=(\varphi \mid \pi(a) \varphi)$ for all $a \in A$. Thus

$$
0=f\left(\left(I-U_{i}^{*}\right) a\right)=\left(\varphi \mid \pi\left(I-U_{i}^{*}\right) \pi(a) \varphi\right)=\left(\left(I-\pi\left(U_{i}\right)\right) \varphi \mid \pi(a) \varphi\right) .
$$

Since $\varphi$ is a cyclic vector, $\pi(A) \varphi$ is dense in $H$. This implies $\left(I-\pi\left(U_{i}\right)\right) \varphi=0$ and hence $\varphi \in \operatorname{ker}\left(I-\pi\left(U_{i}\right)\right)$. Any non-degenerate representation $\pi$ of $C B(\mathcal{H}) \otimes \mathfrak{B}_{\alpha, \beta}^{\varepsilon_{1}, \varepsilon_{2}}$ is of the form $V i d \otimes \pi_{0} V^{*}$, where $\pi_{0}$ is a non-degenerate representation of $\mathfrak{B}_{\alpha, \beta}^{\varepsilon_{1}, \varepsilon_{2}}$. It is easy to check that $\operatorname{ker}\left(\left(i d \otimes \pi_{0}\right)\left(U_{i}\right)-I\right)=\{0\}$, for any $\pi_{0}$. A contradiction. By WN, Proposition 5.1], $U_{1}, U_{2}$ are the Cayley transforms of self-adjoint elements $X_{1} \eta A$ and $X_{2} \eta A$ respectively. Moreover, $D\left(X_{i}\right)=\left(I-U_{i}^{*}\right)\left(C B(\mathcal{H}) \otimes \mathfrak{B}_{\alpha, \beta}^{\varepsilon_{1}, \varepsilon_{2}}\right)$, $i=1,2$.

Proposition 3. $\psi: \mathbb{C}\left[x_{1}, x_{2}\right] \ni x_{i} \rightarrow X_{i} \in\left(C B(\mathcal{H}) \otimes \mathfrak{B}_{\alpha, \beta}^{\varepsilon_{1}, \varepsilon_{2}}\right)^{\eta}$ is a*-homomorphism.

Proof. Let us consider the following set $A_{1,1}=\left[U_{1}, U_{2}\right] A$, where $A=C B(\mathcal{H}) \otimes$ $\mathfrak{B}_{\alpha, \beta}^{\varepsilon_{1}, \varepsilon_{2}}$. Using a simple computation one can show that

$$
\left[U_{1}, U_{2}\right]=P_{3} v \otimes P_{3} v \otimes\left(e_{6}-w_{2}\right)+P_{2} v \otimes P_{2} v \otimes\left(e_{6}-w_{1}\right)
$$

and $Q_{1,1}=P_{3} \otimes P_{3} \otimes\left(\left(e_{6}-w_{2}\right) / 2\right)+P_{2} \otimes P_{2} \otimes\left(\begin{array}{ll}e_{3} & 0 \\ 0 & 0\end{array}\right) \in A$ is the projection of $A$ onto $A_{1,1}$. Now define

$$
D=\left(U_{1}^{*}-I\right)\left(U_{2}^{*}-I\right)\left(I-Q_{1,1}\right) A .
$$


The proposition follows from the following lemma.

Lemma 1. $D$ is dense in $A, D$ is a core for $X_{1}, X_{2}$ and $X_{1} X_{2} a=X_{2} X_{1} a$, for any $a \in D$.

Proof. The proof is similar to that of [S, Lemmas 9.3.2, 9.3.3, 9.3.4]. We begin by showing that $X_{1} X_{2} a=X_{2} X_{1} a$ for any $a \in D$. By definition of the projection $Q_{1,1}$, we have $\left(I-Q_{1,1}\right)\left[U_{1}, U_{2}\right]=\left(I-Q_{1,1}\right)\left[U_{1}-I, U_{2}-I\right]=0$ which implies

$$
\left[U_{1}^{*}-I, U_{2}^{*}-I\right]\left(I-Q_{1,1}\right)=0 \text { and }\left(U_{1}^{*}-I\right)\left(U_{2}^{*}-I\right) b=\left(U_{2}^{*}-I\right)\left(U_{1}^{*}-I\right) b
$$

for any $b \in\left(I-Q_{1,1}\right) A$. Now let $a \in D$. Then

$$
a=\left(U_{1}^{*}-I\right)\left(U_{2}^{*}-I\right) b=\left(U_{2}^{*}-I\right)\left(U_{1}^{*}-I\right) b
$$

with $b \in\left(I-Q_{1,1}\right) A$. Remembering that each element of $D\left(X_{i}\right)$ is of the form $\left(I-U_{i}^{*}\right) a, a \in A, i=1,2$, we see that $a \in D\left(X_{1} X_{2}\right) \cap D\left(X_{2} X_{1}\right)$ and

$$
b=\left(\left(X_{2}-i\right) / 2 i\right)\left(\left(X_{1}-i\right) / 2 i\right) a=\left(\left(X_{1}-i\right) / 2 i\right)\left(\left(X_{2}-i\right) / 2 i\right) a
$$

which implies $X_{2} X_{1} a=X_{1} X_{2} a$ for any $a \in D$.

To prove that $D$ is a core for $X_{1}$ and $X_{2}$ we have to show that $D=\left(I-z_{i}^{*} z_{i}\right)^{1 / 2} D_{i}$, where $D_{i}$ is a dense subset in $A$ and $z_{i}$ is the $z$-transform of $X_{i}, i=1,2$. Let $U_{i}-I=V_{i}\left|U_{i}-I\right|$ be the polar decomposition of $U_{i}-I$ (see [WN, Proposition 0.2]). Then $V_{i} \in M(A)$ and $V_{i}$ is unitary because $\left(U_{i}-I\right) A$ and $\left(U_{i}^{*}-I\right) A$ are dense in $A$. One can check that $\left(I-z_{i}^{*} z_{i}\right)^{1 / 2}=i\left|U_{i}-I\right| / 2=i\left(U_{i}^{*}-I\right) V_{i} / 2$. Since $D=\left(U_{1}^{*}-I\right)\left(U_{2}^{*}-I\right)\left(I-Q_{1,1}\right) A=\left(U_{2}^{*}-I\right)\left(U_{1}^{*}-I\right)\left(I-Q_{1,1}\right) A$, it is sufficient to now show that $\left(U_{i}^{*}-I\right)\left(I-Q_{1,1}\right) A$ is dense in $A$ for $i=1,2$. This follows by the same method as in the proof of Proposition 2 and completes the proof of the lemma and the proposition.

Theorem 6. The functor $F_{\psi}$ is full.

Proof. Let $H_{1}, H_{2}$ be two separable Hilbert spaces and $\pi_{i} \in \operatorname{Rep}\left(\mathfrak{B}_{\alpha, \beta}, H_{i}\right), i=$ 1,2. Then $\tilde{\pi}_{i}=i d_{\mathcal{H}} \otimes \pi_{i}$ is a representation of $C B(\mathcal{H}) \otimes \mathfrak{B}_{\alpha, \beta}^{\varepsilon_{1}, \varepsilon_{2}}, i=1,2$. Let $C$ be a bounded operator intertwining representations $\left(\tilde{\pi}_{1}\left(X_{1}\right), \tilde{\pi}_{1}\left(X_{2}\right)\right)$ and $\left(\tilde{\pi}_{2}\left(X_{1}\right), \tilde{\pi}_{2}\left(X_{2}\right)\right)$, i.e.

$$
C \tilde{\pi}_{1}\left(X_{1}\right) \subseteq \tilde{\pi}_{2}\left(X_{1}\right) C, \quad C \tilde{\pi}_{1}\left(X_{2}\right) \subseteq \tilde{\pi}_{2}\left(X_{2}\right) C
$$

where $\tilde{\pi}_{i}, i=1,2$, are the extensions to affiliated elements. (15) now implies

$$
C \tilde{\pi}_{1}\left(U_{i}\right)=\tilde{\pi}_{2}\left(U_{i}\right) C, \quad C \tilde{\pi}_{1}\left(U_{i}\right)^{*}=\tilde{\pi}_{2}\left(U_{i}\right)^{*} C, i=1,2 .
$$

We have to show that $C=I \otimes A$ where $A \in I\left(\pi_{1}, \pi_{2}\right)$. This follows by direct calculation using simple arguments similar to that of [S, Theorem 9.4.1]. We leave it to the reader.

Corollary 2. There exists a *-homomorphism $\phi: \mathbb{C}\left[x_{1}, x_{2}\right] \rightarrow\left(C B(\mathcal{H}) \otimes C^{*}\left(\mathcal{F}_{2}\right)\right)^{\eta}$ such that the corresponding functor $F_{\phi}$ is full.

Proof. One can easily show that the $C^{*}$-algebra $\mathfrak{B}_{\alpha, \beta}^{\varepsilon_{1}, \varepsilon_{2}}$ is $*$-wild and there exists a *-homomorphism $\varphi: \mathfrak{B}_{\alpha, \beta}^{\varepsilon_{1}, \varepsilon_{2}} \rightarrow M_{n}\left(C^{*}\left(\mathcal{F}_{2}\right)\right), n>0$, which generates the full functor $F_{\varphi}$ from the category $\operatorname{Rep}\left(C^{*}\left(\mathcal{F}_{2}\right)\right)$ into the category $\operatorname{Rep}\left(\mathfrak{B}_{\alpha, \beta}^{\varepsilon_{1}, \varepsilon_{2}}\right)$. Setting $\phi=\varphi \circ \psi$, we obtain that $\phi$ is a $*$-homomorphism from $\mathbb{C}\left[x_{1}, x_{2}\right]$ to $\left(C B(\mathcal{H}) \otimes C^{*}\left(\mathcal{F}_{2}\right)\right)^{\eta}$ and the corresponding functor is full. 
Remark 5. $\psi: C^{*}\left(\mathcal{F}_{2}\right) \ni u_{i} \rightarrow U_{i} \in M\left(C B(\mathcal{H}) \otimes \mathfrak{B}_{\alpha, \beta}^{\varepsilon_{1}, \varepsilon_{2}}\right) \subset\left(C B(\mathcal{H}) \otimes \mathfrak{B}_{\alpha, \beta}^{\varepsilon_{1}, \varepsilon_{2}}\right)^{\eta}$, $i=1,2$, is a $*$-homomorphism and the corresponding functor $F_{\psi}$ is full. However, $U_{1}, U_{2}$ do not generate $C B(\mathcal{H}) \otimes \mathfrak{B}_{\alpha, \beta}^{\varepsilon_{1}, \varepsilon_{2}}$, because $C B(\mathcal{H}) \otimes \mathfrak{B}_{\alpha, \beta}^{\varepsilon_{1}, \varepsilon_{2}}$ is a non-unital $C^{*}$-algebra.

4.2. Representations of the double commutator relations. Consider the *-algebra $\mathfrak{A}=\left\langle x_{1}, x_{2} \mid\left[x_{1},\left[x_{1}, x_{2}\right]\right]=0, x_{i}^{*}=x_{i}, i=1,2\right\rangle$. Clearly, any representation of the commutative algebra $\mathbb{C}\left[x_{1}, x_{2}\right]$ is a representation of the $*$-algebra $\mathfrak{A}$. It follows from the preceding example that non-integrable representations can be complicated, i.e. the problem of unitary classification of such $*$-representations contains as a subproblem the problem of unitary classification of representations of $C^{*}\left(\mathcal{F}_{2}\right)$. In this example we show that the class of representations $\pi$ defined on a domain formed by analytic vectors for $\pi\left(x_{1}\right)$ and $\pi\left(x_{2}\right)$ is $*$-wild.

Let, as before, $\alpha, \beta, \varepsilon_{1}, \varepsilon_{2}>0$ and let $\mathfrak{B}_{\alpha, \beta}^{\varepsilon_{1}, \varepsilon_{2}}$ be the $C^{*}$-algebra which is defined in the first example. On the Hilbert space $H=L_{2}(\mathbb{R}, d x)$ we consider the multiplication operator $q$ by $x$ and the operator of differentiation $p=i \frac{d}{d x}$. Let $a_{1}, a_{2}$ denote the following elements in $M_{3}\left(\mathfrak{B}_{\alpha, \beta}^{\varepsilon_{1}, \varepsilon_{2}}\right)$ :

$$
a_{1}=\left(\begin{array}{lll}
l_{1} e_{1} & 0 & 0 \\
0 & l_{2} e_{1} & 0 \\
0 & 0 & l_{3} e_{1}
\end{array}\right), \quad a_{2}=\left(\begin{array}{lll}
0 & a & b \\
a & 0 & 0 \\
b & 0 & 0
\end{array}\right)
$$

where $l_{i} \in \mathbb{R}$, with $l_{i} \neq l_{j}, i \neq j, e_{n}$ is the unity of $M_{n}\left(\mathfrak{B}_{\alpha, \beta}^{\varepsilon_{1}, \varepsilon_{2}}\right)$. Since any closed operator on $H$ is affiliated with $C B(H)$, we have that $q, p \eta C B(H)$ and there exist uniquely defined self-adjoint elements $X_{1}=e_{3} \otimes q$ and $X_{2}=a_{1} \otimes p+a_{2} \otimes I_{H}$ such that $X_{1}, X_{2} \eta M_{3}\left(\mathfrak{B}_{\alpha, \beta}^{\varepsilon_{1}, \varepsilon_{2}}\right) \otimes C B(H)$. Here $I_{H}$ is the identity operator on $H$.

Proposition 4. $\psi: \mathfrak{A} \ni x_{i} \rightarrow X_{i} \in\left(M_{3}\left(\mathfrak{B}_{\alpha, \beta}^{\varepsilon_{1}, \varepsilon_{2}}\right) \otimes C B(H)\right)^{\eta}$ is a *-homomorphism.

Proof. Let $G$ be the Heisenberg group, i.e., the group of matrices of the form

$$
g=g(t, s, r)=\left(\begin{array}{ccc}
1 & t & r \\
0 & 1 & s \\
0 & 0 & 1
\end{array}\right), t, s, r \in \mathbb{R} .
$$

Then $u: G \rightarrow B(H)$ defined by $u(g(t, 0,0))=e^{i t q}, u(g(0, s, 0))=e^{i s p}, u(g(0,0, r))$ $=e^{i r} I$ is a unitary representation of $G$ in $C B(H)$. By [WN], given a unitary representation $u$ of a real Lie group in a $C^{*}$-algebra $A$, there always exists a dense in $A$ domain $\Phi$ which is invariant with respect to operators of infinitesimal representation of the Lie algebra and is their essential domain. Let $D=M_{3}\left(\mathfrak{B}_{\alpha, \beta}^{\varepsilon_{1}, \varepsilon_{2}}\right) \odot \Phi$. Clearly $D$ satisfies all the required conditions: $D$ is dense in $M_{3}\left(\mathfrak{B}_{\alpha, \beta}^{\varepsilon_{1}, \varepsilon_{2}}\right) \otimes C B(H)$, $D$ is a core for $X_{1}, X_{2}$ and $\left[X_{1},\left[X_{1}, X_{2}\right]\right] a=0$ for any $a \in D$.

We now denote by $R$ the set of all representations $\pi$ of $\mathfrak{A}$ on a Hilbert space $H_{\pi}$ defined on a dense invariant domain consisting of analytic vectors for $\pi\left(x_{1}\right), \pi\left(x_{2}\right)$.

Theorem 7. $R$ is a $*$-wild class of representations.

Proof. Let $i d$ be the identity representation of $M_{3}(C B(H))$ on $H \oplus H \oplus H$. Given a representation $\pi$ of $\mathfrak{B}_{\alpha, \beta}^{\varepsilon_{1}, \varepsilon_{2}},\left((\pi \otimes i d)\left(X_{1}\right),(\pi \otimes i d)\left(X_{2}\right)\right)$ defines a representation which belongs to the class $R$. Here $\pi \otimes i d$ is the unique extension to affiliated elements of $\mathfrak{B}_{\alpha, \beta}^{\varepsilon_{1}, \varepsilon_{2}} \otimes M_{3}(C B(H))=M_{3}\left(\mathfrak{B}_{\alpha, \beta}^{\varepsilon_{1}, \varepsilon_{2}}\right) \otimes C B(H)$. To prove that $R$ is *-wild it is sufficient to show that $X_{1}, X_{2}$ generate $M_{3}\left(\mathfrak{B}_{\alpha, \beta}^{\varepsilon_{1}, \varepsilon_{2}}\right) \otimes C B(H)$. By Theorem [2, the statement will be proved once we prove that $X_{1}, X_{2}$ separate 
representations of $M_{3}\left(\mathfrak{B}_{\alpha, \beta}^{\varepsilon_{1}, \varepsilon_{2}}\right) \otimes C B(H)$ and that $\left(I+X_{2}^{2}\right)^{-1}\left(I+X_{1}^{2}\right)^{-1}\left(I+X_{2}^{2}\right)^{-1} \in$ $M_{3}\left(\mathfrak{B}_{\alpha, \beta}^{\varepsilon_{1}, \varepsilon_{2}}\right) \otimes C B(H)$.

We realize $\mathfrak{B}_{\alpha, \beta}^{\varepsilon_{1}, \varepsilon_{2}}$ as an algebra of operators in a Hilbert space $\mathcal{H}$. Let $F$ be the Fourier transform operator in $H=L_{2}(\mathbb{R}, d x)$. Then $\mathcal{F}=I_{\mathcal{H}}^{3} \otimes F$ is a bounded operator acting on the space $(\mathcal{H} \oplus \mathcal{H} \oplus \mathcal{H}) \otimes H$ and such that $\mathcal{F} X_{1} \mathcal{F}^{-1}=I_{\mathcal{H}}^{3} \otimes p$, $\mathcal{F} X_{1} \mathcal{F}^{-1}=a_{1} \otimes q+a_{2} \otimes I_{H}$ (here $I_{\mathcal{H}}^{3}$ is the identity operator on $\mathcal{H} \oplus \mathcal{H} \oplus \mathcal{H}$ ). The operator $\left(1+q^{2}\right)^{-1}\left(1+p^{2}\right)^{-1}\left(1+q^{2}\right)^{-1}$ is integral with the kernel $K(x, y)=$ $\frac{1}{2}\left(1+x^{2}\right) e^{-|x-y|}\left(1+y^{2}\right)^{-1}$ as it is easy to check. Moreover, this operator is positive with finite trace which implies that it is compact. Therefore,

$$
r=I_{\mathcal{H}}^{3} \otimes\left(I_{H}+q^{2}\right)^{-1}\left(I_{H}+p^{2}\right)^{-1}\left(I_{H}+q^{2}\right)^{-1} \in M_{3}\left(\mathfrak{B}_{\alpha, \beta}^{\varepsilon_{1}, \varepsilon_{2}}\right) \otimes C B(H) .
$$

Let

$$
s=\left(I+\left(a_{1} \otimes q+a_{2} \otimes I\right)^{2}\right)^{-1}\left(I+I \otimes q^{2}\right) .
$$

Clearly, $s$ is bounded. Moreover, $s$ is affiliated with $M_{3}\left(\mathfrak{B}_{\alpha, \beta}^{\varepsilon_{1}, \varepsilon_{2}}\right) \otimes C B(H)$. This is due to the following lemma.

Lemma 2. Let $A$ be a $C^{*}$-algebra, $S \eta A, v \in M(A)$. Assume that $\left[v, z_{S}\right]=\left[v, z_{S}^{*}\right]=$ 0 . Then there exists $T \eta A$ such that $T a=v S a$, for any $a \in D(S)$.

Proof. We shall use Wor1, Theorem 2.3]. Let

$$
a=\left(I-z_{S} z_{S}^{*}\right)^{1 / 2}, b=v z_{S}, c=v z_{S}, d=\left(I-z_{S}^{*} z_{S}\right)^{1 / 2} .
$$

One can easily see that $a, b, c, d \in M(A), a b=c d$, and the sets $a^{*} A=a A$, $d A=d^{*} A$ are dense in $A$.

For

$$
Q=\left(\begin{array}{ll}
d & -c^{*} \\
b & a
\end{array}\right)
$$

we have

$$
Q^{*} Q=\left(\begin{array}{ll}
I-z_{S}^{*} z_{S}+v^{*} v z_{S}^{*} z_{S} & 0 \\
0 & I-z_{S} z_{S}^{*}+v v^{*} z_{S} z_{S}^{*}
\end{array}\right) .
$$

Let $\pi$ be an irreducible representation of $A$ on a Hilbert space $H_{\pi}$, id the canonical representation of $M_{2}(\mathbb{C})$ on $\mathbb{C}^{2}$. Since $\left(I-z_{S}^{*} z_{S}\right)^{1 / 2} A,\left(I-z_{S} z_{S}^{*}\right)^{1 / 2} A$ are dense in $A$ and $v^{*} v z_{S}^{*} z_{S}, v v^{*} z_{S} z_{S}^{*} \geq 0$, one can easily deduce that the range of $(i d \otimes \pi)\left(Q^{*} Q\right)$ is dense in $H_{\pi}$ which implies that $(i d \otimes \pi)(Q)$ is dense in $H_{\pi}$. Using Wor1, Proposition 2.5] we see that $Q(A \oplus A)$ is dense in $A \oplus A$. By Wor1 Theorem 2.3] there exists an element $T \eta A$ such that $d A=\left(I-z_{S}^{*} z_{S}\right)^{1 / 2} A$ is a core of $T$ and $T\left(I-z_{S}^{*} z_{S}\right)^{1 / 2} x=v z_{S} x$ for any $x \in A$. Since $\left(I-z_{S}^{*} z_{S}\right)^{1 / 2} A=D(S)$, $T a=v S a$ for any $a \in D(S)$.

It is known that multipliers are the only bounded elements affiliated with a $C^{*}$ algebra. Therefore $s \in M\left(M_{3}\left(\mathfrak{B}_{\alpha, \beta}^{\varepsilon_{1}, \varepsilon_{2}}\right) \otimes C B(H)\right)$ and srs $\in M_{3}\left(\mathfrak{B}_{\alpha, \beta}^{\varepsilon_{1}, \varepsilon_{2}}\right) \otimes C B(H)$. On the other hand,

$$
\text { srs }=\mathcal{F}\left(I+X_{2}^{2}\right)^{-1}\left(I+X_{1}^{2}\right)^{-1}\left(I+X_{2}^{2}\right)^{-1} \mathcal{F}^{-1}
$$

which yields $\left(I+X_{2}^{2}\right)^{-1}\left(I+X_{1}^{2}\right)^{-1}\left(I+X_{2}^{2}\right)^{-1} \in M_{3}\left(\mathfrak{B}_{\alpha, \beta}^{\varepsilon_{1}, \varepsilon_{2}}\right) \otimes C B(H)$. Since $\mathfrak{B}_{\alpha, \beta}^{\varepsilon_{1}, \varepsilon_{2}}$ is a $*$-wild $C^{*}$-algebra, we conclude that $R$ is a $*$-wild class of representations due to Proposition 1. The fact that $X_{1}, X_{2}$ separate representations follows from [NT] Theorem 3] and the fact that any representation of $M_{3}\left(\mathfrak{B}_{\alpha, \beta}^{\varepsilon_{1}, \varepsilon_{2}}\right) \otimes C B(H)$ is of the 
form $V^{-1}(\pi \otimes i d) V$, where $V$ is a unitary operator, $\pi$ is a representation of $\mathfrak{B}_{\alpha, \beta}^{\varepsilon_{1}, \varepsilon_{2}}$ and $i d$ is the identity representation of $M_{3}(C B(H))$. The proof is finished.

4.3. On unbounded idempotents. Let $B$ be an algebra and let $p_{1}, p_{2}, p_{3}$, $p_{4}$ be idempotents in $B$ such that $p_{1}+p_{2}+p_{3}+p_{4}=0$. Idempotents with this property were studied in [BES]. They arise, in particular, in the study of logarithmic residues in Banach algebras. In [BES] it is shown that non-trivial zero sums of four idempotents do not exist in Banach algebras, however, there are unbounded idempotents in a Hilbert space having this property. Unbounded representations of a $*$-algebra generated by idempotents $p_{1}, p_{2}, p_{3}, p_{4}$ satisfying $p_{1}+p_{2}+p_{3}+p_{4}=0$ were discussed in [ST2]. In this example we shall see that the class of representations defined in [ST2] is $*$-wild. Let $p=\left(p_{1}+p_{2}\right) / 2, q=\left(p_{3}+p_{4}\right) / 2, r=\left(p_{1}-p_{2}\right) / 2$, $s=\left(p_{3}-p_{4}\right) / 2$ (we have $\left.p_{1}=p+r, p_{2}=p-r, p_{3}=q+s, p_{4}=q-s\right)$. Direct computation shows that they satisfy the following relations:

$$
p r=r(1-p), \quad p s=s(-1-p), r^{2}=p(1-p), \quad s^{2}=-p(p+1) .
$$

We assume additionally that

$$
p r^{*}=r p, p s^{*}=s p, p=p^{*},
$$

and denote by $\mathfrak{A}$ the $*$-algebra generated by $p, q, r, s$ and relations ([6)-(17). We define $R \subset \operatorname{Rep}_{\text {unb }}(\mathfrak{A})$ as follows: a family of closed operators $(P, Q, R, S)$ on a Hilbert space $H$ belongs to $R$ iff there exists a linear dense subset $\Phi \subseteq H$ such that $\Phi \subseteq \mathcal{H}_{w}\left(P, Q, R^{*} R, S^{*} S\right), \Phi$ is a core for the operators $R, R^{*}, S$ and $S^{*}$ and relations (6) - (7) hold on $\Phi$. Here $H_{w}(P, Q, R, S)$ denotes the set of analytical vectors for $P, Q, R, S$. It was proved in [ST1 that a subclass $\tilde{R}$ of $R$ defined by the condition ker $P \neq\{0\}$ is manageable, i.e. there exists a $C^{*}$-algebra $A$ and elements $p, q, r, s \eta A$ generating $A$ and such that $\tilde{R}=\{(\pi(p), \pi(q), \pi(r), \pi(s)) \mid$ $\pi \in \operatorname{Rep}(A)\}$. Moreover, all such representations were classified up to a unitary equivalence.

Let $\alpha, \beta>0$. Consider the set $\mathcal{R}$ of all representations $\pi$ of $\mathfrak{S}_{2}$ such that $\|\pi(a)\| \leq \alpha,\|\pi(b)\| \leq \beta$. Denote by $\mathcal{A}_{\alpha, \beta}$ the completion of $\mathfrak{S}_{2} /\{z:\||\|z \mid\|=0\}$ under $\||z|\|=\sup \{\|\rho(z)\| ; \rho \in \mathcal{R}\}$. Let $H$ be a separable infinite-dimensional Hilbert space with an orthonormal basis $\left\{e_{k}\right\}_{k \in \mathbb{Z}}$, and let $P_{k}$ be the orthoprojection onto $\mathbb{C}\left\langle e_{k}\right\rangle, k \in \mathbb{Z}$. We consider operators $v, w$ defined by $v e_{k}=e_{k+1}, v e_{k+1}=e_{k}$ if $k$ is even and $w e_{k}=e_{k+1}, w e_{k+1}=e_{k}$ if $k$ is odd. Clearly, $\left(P_{2 k}+P_{2 k+1}\right) H$ $\left(\left(P_{2 k+1}+P_{2 k+2}\right) H\right)$ is invariant with respect to $v$ (respectively $\left.w\right)$. Now let

$$
\begin{aligned}
\tilde{p} & =\sum_{k \neq 0}(-1)^{k+1} k P_{k} \otimes\left(\begin{array}{cc}
e & 0 \\
0 & e
\end{array}\right), \tilde{q}=\sum_{k \neq 0}(-1)^{k} k P_{k} \otimes\left(\begin{array}{cc}
e & 0 \\
0 & e
\end{array}\right), \\
\tilde{r} & =\left(\sum_{k \neq 0}(2 k+1) v P_{2 k}-2 k v P_{2 k+1}\right) \otimes\left(\begin{array}{cc}
e & 0 \\
0 & e
\end{array}\right)+v P_{0} \otimes\left(\begin{array}{ccc}
e & 0 & 0 \\
0 & 2 e & 0
\end{array}\right), \\
\tilde{s} & =\left(\sum_{k \neq 0}(2 k+1) w P_{2 k+2}-(2 k+2) P_{2 k+1}\right) \otimes\left(\begin{array}{cc}
e & 0 \\
0 & e
\end{array}\right) \\
& +w P_{0} \otimes\left(\begin{array}{ccc}
e & e & a+i b \\
0 & e & e
\end{array}\right) .
\end{aligned}
$$


Here $e$ is the identity element in $\mathcal{A}_{\alpha, \beta}$. We write $\mathcal{H}$ for the Hilbert space $P_{0} H \oplus$ $P_{0} H \oplus P_{0} H \oplus\left(\left(I-P_{0}\right) H \oplus\left(I-P_{0}\right) H\right)$. Direct verification shows that $\tilde{p}, \tilde{q}, \tilde{r}, \tilde{s}$ are affiliated with $C B(\mathcal{H}) \otimes \mathcal{A}_{\alpha, \beta}$ and separate representations of $C B(\mathcal{H}) \otimes \mathcal{A}_{\alpha, \beta}$; moreover, since

$$
\left(I+\tilde{p}^{2}\right)^{-1}=\sum_{k \neq 0}\left(1+k^{2}\right)^{-1} P_{k} \otimes\left(\begin{array}{ll}
e & 0 \\
0 & e
\end{array}\right),\left(I+\tilde{p}^{2}\right)^{-1} \in C B(\mathcal{H}) \otimes \mathcal{A}_{\alpha, \beta} .
$$

Therefore, by Theorem $2 \tilde{p}, \tilde{q}, \tilde{r}, \tilde{s}$ generate the $C^{*}$-algebra $C B(\mathcal{H}) \otimes \mathcal{A}_{\alpha, \beta}$.

Let $D=$ l.s. $\left\{a \otimes b \mid a \in \mathcal{F}, b \in \mathcal{A}_{\alpha, \beta}\right\}$, where $\mathcal{F}$ is the space of finite-dimensional operators in $\mathcal{H}$. Then $D$ is dense in $C B(\mathcal{H}) \otimes \mathcal{A}_{\alpha, \beta}$ and invariant with respect to $\tilde{p}$, $\tilde{q}, \tilde{r}, \tilde{s}, D$ is a core for the elements $\tilde{p}, \tilde{q}, \tilde{r}, \tilde{s}$ and $\tilde{p}, \tilde{q}, \tilde{r}, \tilde{s}$ satisfy relations (6) -(17) on $D$. Moreover, any representation $(\pi(\psi)(p)), \pi(\psi(q)), \pi(\psi(r), \pi(\psi(s)))$ belongs to $R$, where $\psi(x)=\tilde{x}, \pi$ is a representation of $C B(\mathcal{H}) \otimes \mathcal{A}_{\alpha, \beta}$. It implies that the class $R$ is *-wild.

The paper was finished during the author's visit to Université Paris 6, Mathematiques Pures et Appliquees, UFR 920. Their financial support is also acknowledged. I am grateful to Professor N. Th. Varopoulos for his kind hospitality in creating the perfect atmosphere for work during the visit. I wish to thank Professor Yu. Samollenko for many helpful suggestions and discussions during the preparation of the paper.

\section{REFERENCES}

[BES] H.Bart, T.Ehrhardt and B.Silbermann, Zero sums of idempotents in Banach algebras, Integr. Equat. Oper. Th. 19 (1994), 125-134. MR 95c:46071a

[Dal] Yu.L. Daletskii, Continual integrals related to operator evolution equations, Uspekhi Mat. Nauk XVII (1962), no.5, 3-115.

[D] J. Dixmier, $C^{*}$-algebras, North-Holland Publ. Comp., Amsterdam, 1977. MR 56:16388

[DF] P. Donovan, M. R. Freislich, The representation theory of finite graphs and associated algebras, Carleton Mathematical Lecture Notes, No. 5. Carleton University, Ottawa, Ont., 1973, 83 pp. MR 50:9701

[JSW] P.E.T. Jorgensen, L.M. Schmitt, R.F. Werner, Positive representations of general commutation relations allowing Wick ordering, J. Funct. Anal. 134 (1995), no. 1, 33-99. MR 96h:81033

[KS] S. A. Kruglyak and Yu. S. Samollenko, On complexity of description of representation of *-algebras generated by idempotents, Proc. Amer. Math. Soc., 128 (2000), no. 6, 16551664. MR 2000j:46099

[NT] L. P. Nizhnik and L. B. Turowska, Representations of double commutator by matrixdifferential operators, Methods Funct. Anal. Topol. 3 (1997), no. 3, 75-80. MR 2001e:47043

[OS] V. Ostrovskiı̌, Yu. Samollenko, Introduction to the Theory Representation of Finitely Presented $*$-algebras. 1. Representations by bounded operators. Rev. Math. Math. Phys. 11 (1999), part 1, 1-261.

[S] V. V. Sergeichuk, Unitary and Euclidean representations of a quiver, Linear Algebra Appl. 278 (1998), no. 1-3, 37-62. MR 99g:16020

[Pat] A. L. T. Paterson, The class of locally compact groups $G$ for which $C^{*}(G)$ is amenable. Harmonic Analysis, Luxembourg, 1987, Lecture Notes in Mathematics, Vol. 1359, New York, 1988. MR 90m:22019

[Ped] G. K. Pedersen, $C^{*}$-algebras and their Automorphism Groups, Academic Press, London, 1979.

[ST1] Yu. S. Samollenko and L. B. Turowska, On representations of $*$-algebras by unbounded operators, Funkt. Anal. Prilozh. 31 (1997), no. 4, 80-83.

[ST2] Yu. S. Samollenko and L. B. Turowska, On bounded and unbounded idempotents whose sum is a multiple of the identity, Preprint 2001-44, Chalmers University of Technology. 
[S] K. Schmüdgen, Unbounded operator algebras and representation theory, Akademie-Verlag, Berlin, 1990.

[Wor1] S. L. Woronowicz, Unbounded elements affiliated with $C^{*}$-algebras and non-compact quantum groups, Commun. Math. Phys. 136 (1991), 399-432. MR 92b:46117

[WN] S. L. Woronowicz and K. Napiorkowski, Operator theory in the $C^{*}$-algebra framework, Rep. Math. Phys. 31 (1992), 353-371. MR 94k:46123

[Wor2] S. L. Woronowicz, $C^{*}$-algebras generated by unbounded elements, Reviews in Mathematical Physics 7 (1995), no. 3, 481-521. MR 96c:46056

Department of Mathematics, Chalmers University of Technology, 41296 Göteborg, SWEDEN

E-mail address: turowska@math.chalmers.se 